Original Article

\title{
Sesamin induces A549 cell mitophagy and mitochondrial apoptosis via a reactive oxygen species-mediated reduction in mitochondrial membrane potential
}

\author{
Shasha Yang ${ }^{1}$, Xiangdan $\mathrm{Li}^{1, *}$, Haowen Dou ${ }^{1}$, Yulai Hu${ }^{1}$, Chengri Che ${ }^{2}$, and Dongyuan $\mathrm{Xu}^{1, *}$ \\ ${ }^{1}$ Center of Morphological Experiment, Medical College of Yanbian University, ${ }^{2}$ Department of Thoracic Surgery, Affiliated Hospital of Yanbian University, Yanji, \\ Jilin 133000, China
}

\section{ARTICLE INFO}

Received October 27, 2019

Revised February 28, 2020

Accepted February 28, 2020

*Correspondence

Xiangdan Li

E-mail: lixiangdan@ybu.edu.cn

Dongyuan Xu

E-mail: dyxu@ybu.edu.cn

\section{Key Words}

Apoptosis

Lung cancer

Mitophagy

Reactive oxygen species

Sesamin
ABSTRACT Sesamin, a lipid-soluble lignin originally isolated from sesame seeds, which induces cancer cell apoptosis and autophagy. In the present study, has been reported that sesamin induces apoptosis via several pathways in human lung cancer cells. However, whether mitophagy is involved in sesamin induced lung cancer cell apotosis remains unclear. This study, the anticancer activity of sesamin in lung cancer was studied by reactive oxygen species (ROS) and mitophagy. A549 cells were treated with sesamin, and cell viability, migration ability, and cell cycle were assessed using the CCK8 assay, scratch-wound test, and flow cytometry, respectively. ROS levels, mitochondrial membrane potential, and apoptosis were examined by flow cytometric detection of DCFH-DA fluorescence and by using JC-1 and TUNEL assays. The results indicated that sesamin treatment inhibited the cell viability and migration ability of $A 549$ cells and induced $G_{0} / G_{1}$ phase arrest. Furthermore, sesamin induced an increase in ROS levels, a reduction in mitochondrial membrane potential, and apoptosis accompanied by an increase in cleaved caspase-3 and cleaved caspase-9. Additionally, sesamin triggered mitophagy and increased the expression of PINK1 and translocation of Parkin from the cytoplasm to the mitochondria. However, the antioxidant $\mathrm{N}$-acetyl-L-cysteine clearly reduced the oxidative stress and mitophagy induced by sesamin. Furthermore, we found that cyclosporine A (an inhibitor of mitophagy) decreased the inhibitory effect of sesamin on A549 cell viability. Collectively, our data indicate that sesamin exerts lethal effects on lung cancer cells through the induction of ROS-mediated mitophagy and mitochondrial apoptosis.

\section{INTRODUCTION}

Lung cancer is a major cause of morbidity and mortality worldwide and is one of the leading causes of cancer-related deaths [1]. Non-small-cell lung cancer (NSCLC) is a type of lung cancer that includes squamous carcinoma, adenocarcinoma, and large-cell carcinoma. Compared with small-cell carcinoma, NSCLC cells grow more slowly and metastasize relatively late. NSCLC accounts for approximately $80 \%$ to $85 \%$ of the total number of lung cancer cases and is currently treated mainly using chemotherapy [2]. However, severe toxicity greatly limits chemotherapy. Therefore, there is an urgent need to develop a safe and effective adjuvant therapy for NSCLC.

Sesamin is a lipid-soluble lignin that was first found in sesame seeds and has since been identified in 30 different species of plants. Detailed studies have shown that sesamin has anticancer effects, which are mainly attributable to its anti-proliferative, anti-metastatic, anti-inflammatory, pro-apoptotic, and pro-

\section{(i) (5)}

This is an Open Access article distributed under the terms of the Creative Commons Attribution Non-Commercial License, which permits unrestricted non-commercial use, distribution, and reproduction in any medium, provided the original work is properly cited. Copyright $\odot$ Korean J Physiol Pharmacol, pISSN 1226-4512, elSSN 2093-3827
Author contributions: X.L. and D.X. designed experiments; S.Y., X.L. and H.D. carried out experiments; S.Y., Y.H. and C.C. analyzed experimental results; S.Y. and D.X. wrote the manuscript; X.L. and D.X. revising the manuscript; D.X. approved the manuscript. 
autophagocytic properties [3]. In recent years, several studies have reported the anticancer effects of sesamin on a variety of cancers, such as breast, prostate, cervical, and lung cancer [4-6]. Sesamin was reported to induce apoptosis in human lung cancer cells through inhibition of the mitochondrial protease Lon and activation of the DNA-damage checkpoints [7]. Recent research suggested that sesamin blocks the phosphoinositide 3-kinase (PI3K)/ Akt signaling pathway in lung cancer by downregulation of the expression of cyclooxygenase 2 (COX2), leading to cell cycle arrest and apoptosis [8]. A recent study reported that sesamin suppressed NSCLC cell proliferation and induced G1 arrest of the cell cycle and apoptosis via the Akt/p53 pathway [9]. Although previous studies have confirmed that sesamin can inhibit proliferation and promote the apoptosis of NSCLC, the mechanism is still not completely clear. Our has previously reported that sesamin could induce autophagic death in cervical cancer cells [6]; therefore, we hypothesized that mitochondrial autophagy may be involved in the inhibitory effect of sesamin on lung cancer.

Oxidative phosphorylation of mitochondria is an efficient process that is accompanied by excessive reactive oxygen species (ROS) production [10]. Excessive ROS in the body damages mitochondria and promotes the release of apoptosis-promoting factors, eventually leading to cell death [11]. To maintain mitochondrial homeostasis, damaged mitochondria need to be removed in time to promote mitochondrial renewal. Mitochondrial renewal in cells is mainly through the selective autophagy pathway. In this process, the dysfunction and aging mitochondria are selectively degraded, which is called mitophagy [12]. The early manifestation of mitophagy is mitochondrial depolarization, after which mitochondria are specifically enveloped in autophagosomes, which then fuse with lysosomes to complete the degradation and renewal of mitochondria [13]. Mitochondrial membrane potential collapse is a prerequisite and early event for the PINK1/Parkin pathway activation and mitophagy. When the mitochondrial membrane potential disintegrates, PINK1 stably accumulates on the mitochondrial outer membrane, activating the transfer of Parkin from the cytoplasm to the mitochondria, and eventually inducing mitophagy [14].

In this study, we used the A549 cell line to investigate the inhibitory effect of sesamin on NSCLC. We provide evidence to support the therapeutic role of sesamin in lung cancer through induction of oxidative stress-mediated apoptosis and mitophagy, further confirming its anticancer properties.

\section{METHODS}

\section{Cell culture}

The A549 lung adenocarcinoma cell line was obtained from the Cell Resource Center of the Shanghai Institute of Life Sciences, Chinese Academy of Sciences. Cells were cultured in complete growth medium consisting of Dulbecco's modified Eagle's medium (DMEM; Gibco, Carlsbad, CA, USA) supplemented with $10 \%$ fetal bovine serum (FBS; Gibco), $100 \mathrm{~g} / \mathrm{ml}$ streptomycin, and $100 \mathrm{U} / \mathrm{ml}$ penicillin at $37^{\circ} \mathrm{C}$ in a humidified $5 \% \mathrm{CO}_{2}$ and $95 \%$ air atmosphere.

\section{Cell viability assay}

Cell viability was determined using the Cell Counting Kit-8 (CCK-8; Dojindo, Kumamoto, Japan) as described by the manufacturer. Briefly, $2 \times 10^{3}$ cells per well were plated in 96-well culture plates and incubated in $100 \mu \mathrm{l}$ of complete growth medium. The synergistic effects of sesamin on human lung cancer A549 cell line viability were assessed by treating cells with $10,20,40$, 60,80 , and $100 \mu \mathrm{M}$ sesamin (purity > 98\%; Chengdu Must Bio, Chengdu, China) dissolved in chloroform (Amresco, Solon, $\mathrm{OH}$, USA) for $12,24,36$, and $48 \mathrm{~h}$; cells treated with vehicle $(0.1 \%$ chloroform) were used as controls. After treatment, $10 \mu \mathrm{l} \mathrm{CCK-8}$ solution was added to each well and plates were incubated for an additional $4 \mathrm{~h}$ at $37^{\circ} \mathrm{C}$. The percentage of viable cells was determined by measuring optical density in triplicate wells at $450 \mathrm{~nm}$ using a microplate reader (Molecular Devices, Sunnyvale, CA, USA).

\section{Scratch-wound test}

The effects of sesamin on the migration of A549 cells was evaluated using the scratch-wound test. For this test, $2 \times 10^{5}$ cells per well were seeded in 6-well plates, and then incubated overnight to allow cells to cover the entire plate. Thereafter, a "wound" was made by scratching the monolayer, and plates were washed three times with phosphate-buffered saline (PBS). Cells were then treated with 20 or $40 \mu \mathrm{M}$ sesamin, or vehicle $(0.1 \%$ chloroform), in DMEM for $48 \mathrm{~h}$. Images were acquired at 0 and $48 \mathrm{~h}$ using an inverted microscope at $200 \times$ magnification. The wound-closure rate was calculated according to the formula, ([0 h scratch width - 48 h scratch width] / [0 h scratch width]) $\times 100 \%$.

\section{Transmission electron microscopy}

A549 cells were seeded in 6-well plates and cultured in DMEM containing $40 \mu \mathrm{M}$ sesamin or $0.1 \%$ chloroform (vehicle control) at $37^{\circ} \mathrm{C}$ and $5 \% \mathrm{CO}_{2}$ for $48 \mathrm{~h}$. Cells were collected by centrifugation at $300 \times \mathrm{g}$ for $10 \mathrm{~min}$ at room temperature and fixed in $2.5 \% \mathrm{glu}$ taraldehyde for $1 \mathrm{~h}$. Next, cells were fixed in a $1 \%$ osmium tetroxide solution for $1 \mathrm{~h}$, dehydrated with a graded series of ethanol washes $(30 \%, 60 \%, 70 \%, 90 \%$, and $100 \%)$, which infiltrated the cells, and then embedded in LR White resin. Ultrathin sections (50-75 nm), sliced with an LKB ultramicrotome, were stained with $2 \%$ aqueous uranyl acetate and $2 \%$ aqueous lead citrate, and transmission electron microscopy (TEM) images were obtained at 5,000× magnification. 


\section{Terminal deoxynucleotidyl transferase dUTP nick-end labeling (TUNEL) assay}

Apoptotic DNA fragmentation was detected by fluorescence microscopy using a DeadEnd Fluorometric TUNEL System kit (Promega, Wisconsin, WI, USA). A549 cells were seeded at $1 \times$ $10^{5}$ cells/well in 6-well plates and grown in a humidified atmosphere of $5 \% \mathrm{CO}_{2}$ at $37^{\circ} \mathrm{C}$. After treating cells with $40 \mu \mathrm{M}$ sesamin and/or the ROS scavenger N-acetyl-L-cysteine (NAC, $5 \mu \mathrm{M}$; Sigma-Aldrich) for $48 \mathrm{~h}$, TUNEL assays were performed according to the manufacturer's instructions. Samples were immediately analyzed under a fluorescence microscope using standard filter sets for fluorescein (green) at $520 \pm 20 \mathrm{~nm}$ and propidium iodide (PI; red) at $>620 \mathrm{~nm}$.

\section{Cell Mitochondria Isolation}

Cell Mitochondria Isolation and cytoplasmic protein extraction were measured using a Cell Mitochondria Isolation Kit (Beyotime, Shanghai, China) as described by the manufacturer. Briefly, cells were collected by centrifugation at $100 \mathrm{~g}$ after digestion with trypsin cell digestive solution, and precipitated with cold PBS and then centrifuged at $600 \mathrm{~g}$ and $4^{\circ} \mathrm{C}$ for $5 \mathrm{~min}$. After discarding the supernatant, add $1 \mathrm{ml}$ of mitochondrial separation reagent to $2 \times$ $10^{7}$ cells, gently suspend the cells and place them in ice bath for 10 min. Next, transfer the cell suspension to a glass homogenizer of appropriate size for about 30 times. The cell homogenate was centrifuged at $600 \mathrm{~g}$ and $4^{\circ} \mathrm{C}$ for $10 \mathrm{~min}$. The supernatant was then transferred to another centrifuge tube and centrifuged at 11,000 $\mathrm{g}$ for $10 \mathrm{~min}$ at $4^{\circ} \mathrm{C}$. The precipitate is the isolated cell mitochondria. Carefully collect the supernatant and centrifugate it at $4^{\circ} \mathrm{C}$ for $10 \mathrm{~min}$ and he supernatant is cytoplasmic protein (mitochondria removed).

\section{Western blotting}

Treated cells were washed twice with ice-cold PBS (pH 7.4) and lysed in RIPA buffer (Solarbio, Beijing, China) supplemented with phenylmethylsulfonyl fluoride (PMSF; Solarbio) and a phosphatase inhibitor (Roche, Basel, Switzerland). Lysates were incubated for $5 \mathrm{~min}$ on ice and then microcentrifuge at 14,000 rpm for 10 $\min$ at $4^{\circ} \mathrm{C}$. Protein concentration in whole-cell lysates was quantified using a BCA kit (Mbchem, Shanghai, China), resolved by sodium dodecyl sulfate polyacrylamide gel electrophoresis (SDSPAGE), and then transferred to polyvinylidene difluoride (PVDF) membranes (Millipore, Bedford, MA, USA). Non-specific binding was blocked by incubating membranes with TBST (Trisbuffered saline with $0.1 \%$ Tween-20) containing $5 \%$ non-fat milk for $2 \mathrm{~h}$ at room temperature. Thereafter, membranes were incubated overnight at $4^{\circ} \mathrm{C}$ with primary antibodies (diluted in TBST) against cleaved caspase-3, cleaved caspase- 9 , cytochrome c (1:1,000; all from Cell Signaling, Beverly, MA, USA); and Parkin
(1:1,000), PINK1 (1:2,000), Bax (1:1,000), B-cell lymphoma-2 (Bcl2) $(1: 1,000), \beta$-Tubulin $(1: 2,000), \operatorname{VDAC1}(1: 2,000)$ and GAPDH (1:5,000, internal control; all from Santa Cruz Biotechnology, Santa Cruz, CA, USA). Membranes were then washed and incubated with appropriate horseradish peroxidase-conjugated secondary antibodies (Santa Cruz Biotechnology) for $1 \mathrm{~h}$ at room temperature. Finally, protein signals were detected using enhanced chemiluminescence (ECM) (Western Blotting Kit; Boster, Wuhan, China).

\section{Measurement of mitochondrial membrane potential}

Mitochondrial membrane potential $(\Delta \psi \mathrm{m})$ was measured using a JC-1 assay kit (Beyotime) as described by the manufacturer. Briefly, A549 cells were seeded at a density of $5 \times 10^{5}$ per well in 6-well plates, incubated for $12 \mathrm{~h}$, and then treated with $40 \mu \mathrm{M}$ sesamin and/or $5 \mathrm{mM} \mathrm{NAC} \mathrm{for} 48 \mathrm{~h}$. Following removal of the culture medium, $1 \mathrm{ml}$ of cell culture medium and $1 \mathrm{ml}$ of JC-1 dye buffer were added, fully mixed, and incubated at $37^{\circ} \mathrm{C}$ for $20 \mathrm{~min}$. The supernatant was removed and cells were washed twice with JC-1 dye buffer (1x). Washed cells were resuspended in culture medium $(2 \mathrm{ml})$ and observed under a fluorescence microscope.

\section{Intracellular ROS measurement}

A549 cells were plated in 6-well plates and incubated for $12 \mathrm{~h}$ to allow cells to reach the exponential growth phase. Cells were then treated with $40 \mu \mathrm{M}$ sesamin (or $0.1 \%$ chloroform) and/or 5 $\mathrm{mM} \mathrm{NAC}$, at $37^{\circ} \mathrm{C}$ and $5 \% \mathrm{CO}_{2}$ for $48 \mathrm{~h}$, after which they were harvested by centrifugation for $5 \mathrm{~min}$ at $37^{\circ} \mathrm{C}$ and resuspended in DCFH-DA $(10 \mu \mathrm{M})$ for $30 \mathrm{~min}$ at $37^{\circ} \mathrm{C}$ in the dark. The intracellular $\mathrm{H}_{2} \mathrm{O}_{2}$ content was determined by flow cytometry (BD Biosystems, Franklin lake, NJ, USA) and analyzed using Cell Quest software (BD Biosystems).

\section{Flow cytometry}

Apoptosis was examined by staining cells with an Annexin V Apoptosis detection kit I (BD Biosciences, San Jose, CA, USA) as described by the manufacturer. Briefly, cells were washed twice with cold PBS and resuspended in $1 \times$ Binding Buffer at a concentration of $1 \times 10^{6}$ cells $/ \mathrm{ml}$. Subsequently, $100 \mu$ l cell solution $(1 \times$ $10^{5}$ cells) was mixed with $5 \mu \mathrm{l}$ Annexin V and $5 \mu \mathrm{PI}$ and incubated for $15 \mathrm{~min}$ at room temperature in the dark. Finally, $400 \mu \mathrm{l}$ of $1 \times$ Binding Buffer was added to each tube, and apoptosis was analyzed within $1 \mathrm{~h}$ using a FACScan flow cytometer (BD Biosystems).

Cell cycle was determined with a cell cycle detection kit (Beyotime) according to the manufacturer's protocol. In brief, A549 cells were washed twice with cold PBS and fixed with $70 \%$ ethanol at $4^{\circ} \mathrm{C}$ for $2 \mathrm{~h}$, Following PBS washing for 3 times and $30 \mathrm{~min}$ of PI staining at $37^{\circ} \mathrm{C}$. Finally, cell cycle was analyzed within $1 \mathrm{~h}$ 
using a FACScan flow cytometer (BD Biosystems).

\section{Statistical analysis}

The data presented are expressed as means \pm standard deviation (SD) of three independent experiments performed in triplicate. The significance of differences between two means was determined by Student's t-test using SPSS 19.0 software (IBM Co., Armonk, NY, USA). p-values $<0.05$ were considered statistically significant.

\section{RESULTS}

\section{Sesamin inhibits the proliferation and migration of human lung cancer cells}

A549 cells were treated with $10,20,40,60,80$, and $100 \mu \mathrm{M}$ sesamin, or $0.1 \%$ chloroform (vehicle control), for $12,24,36$, and $48 \mathrm{~h}$, and cell viability was assessed using CCK- 8 assays. These analyses showed that sesamin exerted a time- and concentration- dependent depressant effect on the proliferation of A549 cells $(\mathrm{p}<0.05)$ (Fig. 1A). The effect of sesamin on the migration ability of A549 cells was assessed by treating cells with 20 or $40 \mu \mathrm{M}$ sesamin (or $0.1 \%$ chloroform) for 24 and $48 \mathrm{~h}$, and then performing scratch-wound assays. These experiments showed that the migratory ability of sesamin-treated A549 cells, measured as the degree of wound closure, was lower than that of the control group (Fig. 1B). A549 cells were treated with $40 \mu \mathrm{M}$ sesamin (or $0.1 \%$ chloroform) for $48 \mathrm{~h}$, and then detected the cell cycle by flow cytometry, the results showed that the cells in G2 and S phase decreased and blocked in $G_{0} / G_{1}$ phase after sesamin treatment (Fig. 1C), further indicating the suppressive effect of sesamin on the proliferation and migration of A549 cells.

\section{Sesamin triggers the collapse of mitochondrial membrane potential in A549 cells by increasing ROS levels}

To establish whether sesamin stimulates intracellular oxidative stress, we examined ROS levels based on DCFH-DA fluorescence using flow cytometry. As shown in Fig. 2A, $40 \mu \mathrm{M}$ sesamin sig-

A

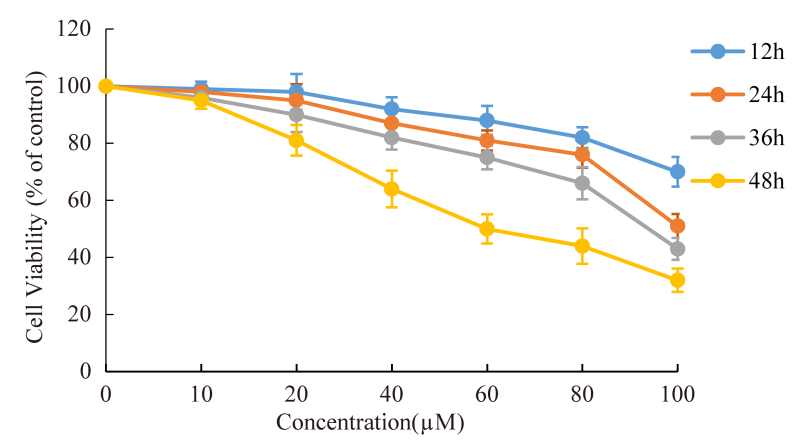

B

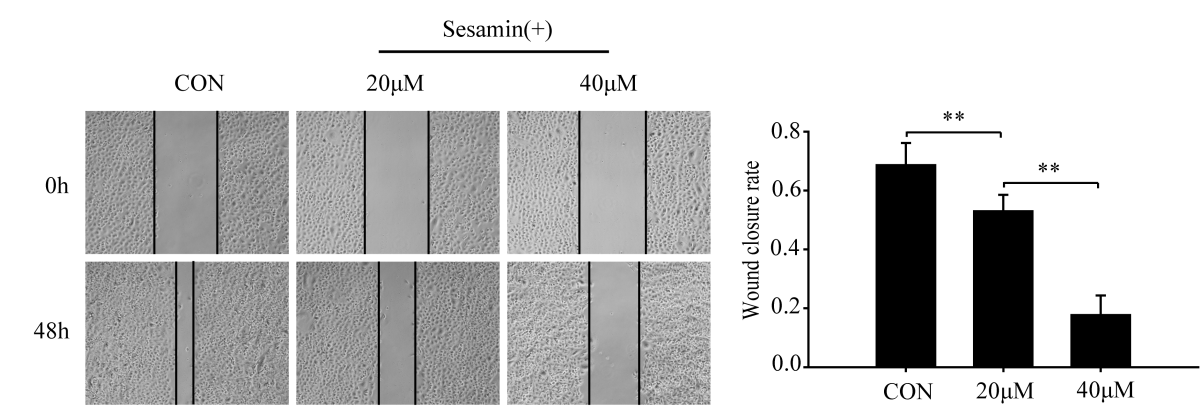

C
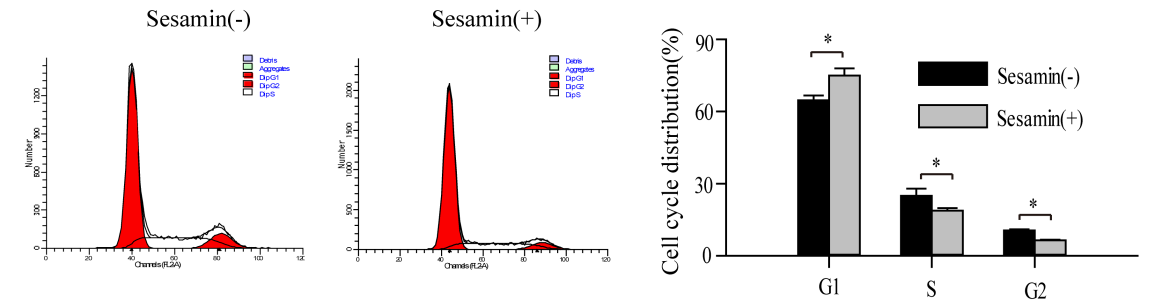

Fig. 1. Sesamin inhibits the proliferation and migration of human lung cancer cells. (A) A549 cells were treated with $10,20,40,60,80$, and $100 \mu \mathrm{M}$ sesamin or $0.1 \%$ chloroform (as a control) for $12,24,36$, and $48 \mathrm{~h}$, and cell viability was examined using CCK-8 assays. Data are means \pm standard deviation (SD) $(\mathrm{n}=$ 3). (B) Scratch-wound assays were used to assess the effect of sesamin on the migration ability of A549 cells treated with 20 or $40 \mu \mathrm{M}$ sesamin (or $0.1 \%$ chloroform) for $48 \mathrm{~h}$. Quantitative analysis of healing rate in wound-scratch assays. Data are means \pm SD $\left(n=3 ;{ }^{* *} p<0.01\right)$. (C) A549 cells were treated with $40 \mu \mathrm{M}$ sesamin (or $0.1 \%$ chloroform) for $48 \mathrm{~h}$, and then detected the cell cycle by flow cytometry. Data are means $\pm S D(n=3$; $\left.{ }^{*} \mathrm{p}<0.05\right)$. 
nificantly increased ROS generation in A549 cells. This increase was significantly attenuated by pretreatment of A549 cells with the antioxidant NAC $(5 \mathrm{mM} ; \mathrm{p}<0.05)$. It is well known that ROS accumulation can cause mitochondrial damage. Accordingly, we measured changes in mitochondrial membrane potential using a JC-1 assay kit containing the cationic dye, which undergoes a

A

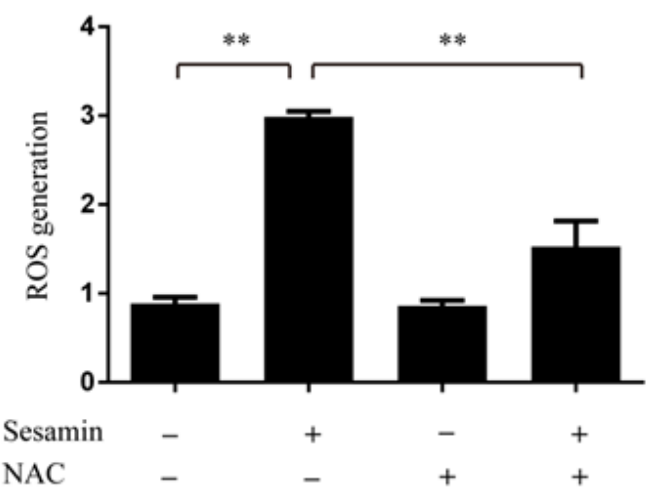

B

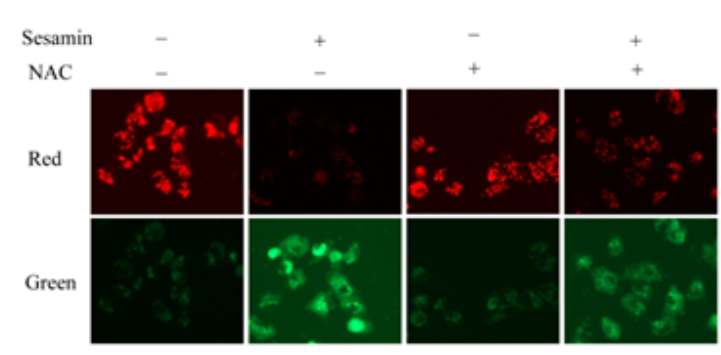

\section{C}

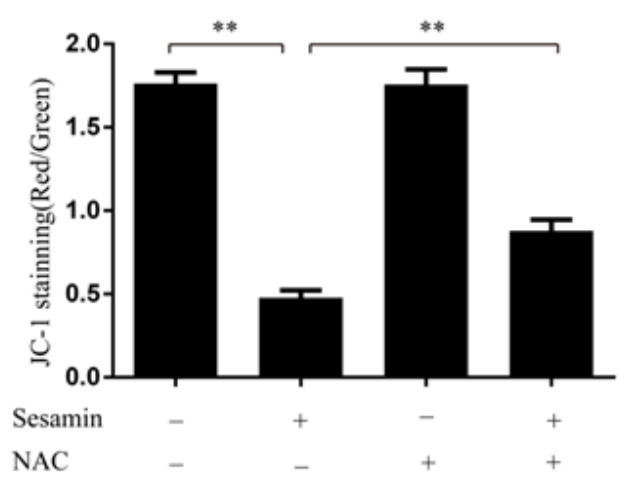

Fig. 2. Sesamin triggers the collapse of mitochondrial membrane potential by increasing reactive oxygen species (ROS) levels in A549 cells. (A) A549 cells were treated with $40 \mu \mathrm{M}$ sesamin and/or 5 mM NAC for $48 \mathrm{~h}$, and then ROS levels were examined by flow cytometric detection of DCFH-DA fluorescence. Data are means \pm standard deviation (SD) $(n=3$; **p $<0.01)$. (B) Changes in mitochondrial membrane potential were detected a JC-1 assay kit containing the cationic dye, which undergoes a readily detectable shift from red to green with decreases in membrane potential. The images were obtained at $400 x$ magnification. (C) Quantitative analysis of JC-1 results. Data are means $\pm \mathrm{SD}\left(\mathrm{n}=3 ;{ }^{* *} \mathrm{p}<0.01\right)$. NAC, N-acetyl-L-cysteine. readily detectable shift from red to green with decreases in membrane potential. As shown in Fig. $2 \mathrm{~B}$ and $\mathrm{C}$, sesamin treatment increased the green/red fluorescence ratio in A549 cells, an effect

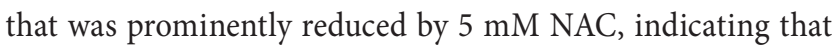
sesamin triggers the collapse of mitochondrial membrane potential through ROS accumulation.

\section{Sesamin acts through a ROS-dependent mitochondrial pathway to induce apoptosis of A549 cells}

To investigate whether sesamin induces A549 cell apoptosis, we used flow cytometry and TUNEL staining. A549 cells were treated with $40 \mu \mathrm{M}$ sesamin for $48 \mathrm{~h}$, after which apoptosis was assessed by flow cytometric detection of TUNEL-stained cells. These experiments showed that sesamin promoted A549 cell apoptosis, and that $5 \mathrm{mM} \mathrm{NAC}$ alleviated this effect (Fig. 3A-D). Next, we assessed the expression of the mitochondrial apoptosisrelated proteins, cleaved caspase-3, cleaved caspase-9, Bax, Bcl-2, and cytochrome $\mathrm{c}$ by Western blotting. This analysis showed that sesamin treatment increased the expression of cleaved caspase-3, cleaved caspase-9, cytochrome $\mathrm{c}$ and the ratio of $\mathrm{Bax} / \mathrm{Bcl}-2$ in A549 cells; notably, these increases were block by pretreatment with $5 \mathrm{mM} \mathrm{NAC} \mathrm{(Fig.} \mathrm{3E,} \mathrm{F).} \mathrm{Taken} \mathrm{together,} \mathrm{our} \mathrm{data} \mathrm{suggest}$ that sesamin induces A549 cell apoptosis via a ROS-dependent mitochondrial pathway.

\section{Sesamin triggers mitophagy in A549 cells through a ROS-mediated PINK1/Parkin pathway}

Decreases in mitochondrial membrane potential may lead to mitophagy, a process that involves the PINK1/Parkin pathway $[15,16]$. To investigate whether sesamin triggers mitophagy, we used TEM to examine ultrastructural changes in A549 cells treated with $40 \mu \mathrm{M}$ sesamin for $48 \mathrm{~h}$. As shown in Fig. 4A, mitochondrial swelling and degeneration, and numerous autophagosomes containing mitochondria, were observed under the electron microscope, confirming that sesamin treatment induces mitophagy. To further investigate effect of sesamin on mitophagy, we next examined changes of PINK1 in whole cell and Parkin in mitochondria and cytoplasm.by Western blotting. As shown in Fig. $4 \mathrm{~B}, \mathrm{C}$, sesamin increased the expression of PINK1 in whole cell and Parkin in mitochondria, but decreased the level of Parkin in cytoplasm, indicating the translocation of Parkin from cytoplasm to mitochondria. In addition, NAC blocked the up-regulation of PINK1 and the translocation of Parkin induced by sesamin, indicating a role for ROS in the induction of mitophagy by sesamin.

\section{Inhibition of mitophagy weakens the inhibitory effect of sesamin on A549 cell viability}

Cyclosporine A (CsA), a specific inhibitor of mitophagy, is 
A

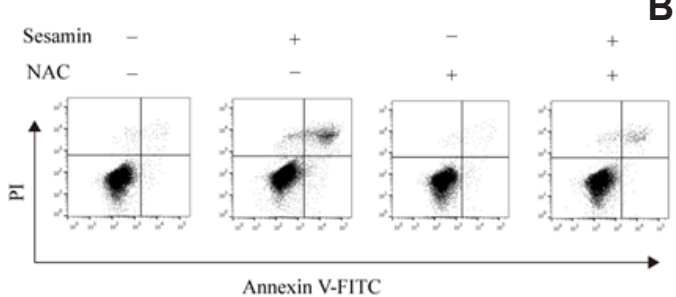

C

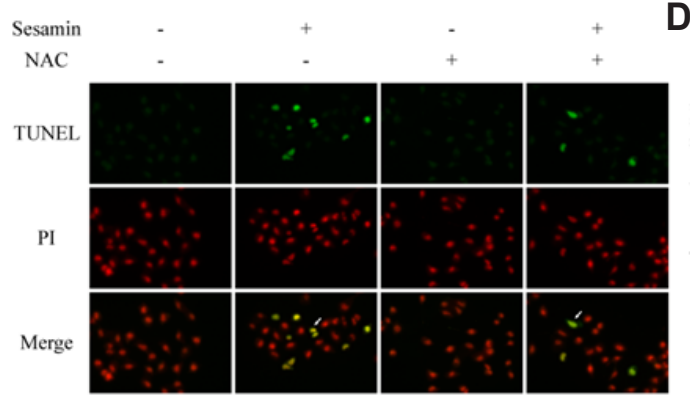

D
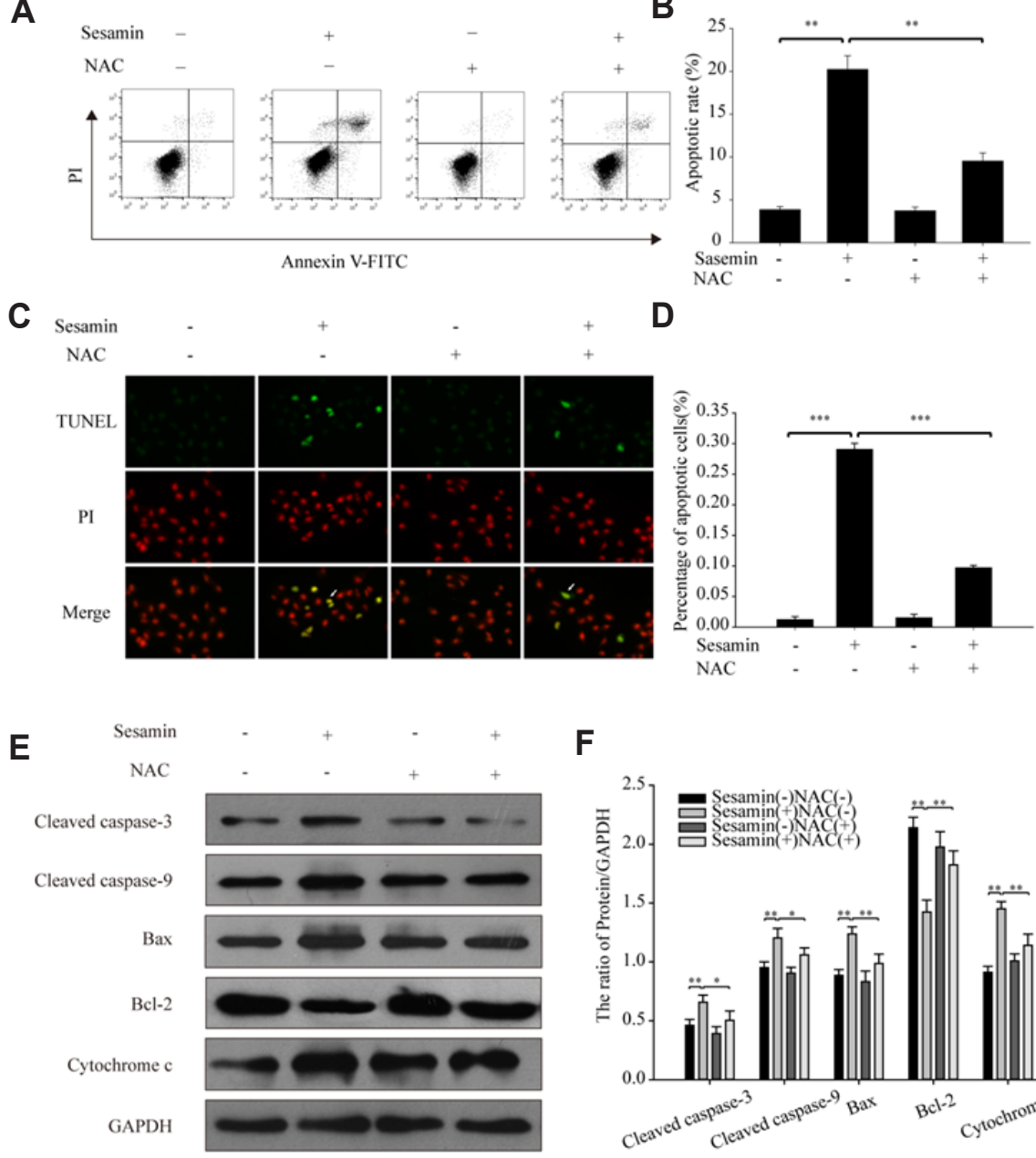

F

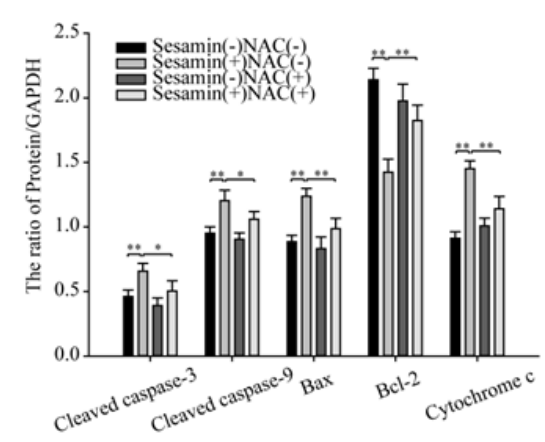

Fig. 3. Sesamin induces A549 cell apoptosis via a reactive oxygen species (ROS)-dependent mitochondrial pathway. (A) A549 cells were treated with $40 \mu \mathrm{M}$ sesamin and/or $5 \mathrm{mM} \mathrm{N}$ acetyl-L-cysteine (NAC) for $48 \mathrm{~h}$, stained with Annexin V-FITC and propidium iodide (PI), and then analyzed using a FACScan flow cytometer. (B) Summary data for Annexin V-FITC/PI staining. Data are means \pm standard deviation (SD) $(\mathrm{n}=$ $3{ }^{* *} \mathrm{p}<0.01$ ). (C) A549 cells were treated with $40 \mu \mathrm{M}$ sesamin and/or $5 \mathrm{mM}$ NAC for $48 \mathrm{~h}$, and TUNEL assays were performed according to the manufacturer's instructions. Samples were analyzed under a fluorescence microscope using standard filter sets for fluorescein (green) and propidium iodide ( $\mathrm{Pl}$; red). The images were obtained at $200 x$ magnification. (D) Summary data for TUNEL assays. Data are means \pm SD $(n=$ $\left.3 ;{ }^{* * *} \mathrm{p}<0.001\right)$. (E) Expression of the mitochondrial apoptosis-related proteins, caspase-3, caspase-9, Bax, Bax/B-cell lymphoma-2 (Bcl-2) and cytochrome $\mathrm{C}$ in A549 cells treated with $40 \mu \mathrm{M}$ sesamin and/or $5 \mathrm{mM} \mathrm{NAC}$ for $48 \mathrm{~h}$ was assessed by Western blot analysis. (F) Quantification of caspase-3, caspase-9, Bcl-2 and cytochrome c. Data are means \pm SD $(n=$ $\left.3 ;{ }^{*} p<0.05,{ }^{* *} p<0.01\right)$. capable of inhibiting mitochondrial depolarization and mitochondrial autophagosome formation by interfering with the interaction of cyclophilin D with the mitochondrial permeability transition pore [17]. To explore the role of mitophagy in sesamintreated A549 cells, we blocked mitophagy with $5 \mu \mathrm{M}$ CsA and detected the expression of PINK1 in whole cell and Parkin in mitochondria and cytoplasm by Western blotting. This analysis showed that CsA inhibited the sesamin-induced increases in PINK1 in whole cell and Parkin in mitochondria. In addition, compared with the sesamin treated group, the expression of Parkin in the cytoplasm (sesamin and CsA combined treatment group) increased, indicating Parkin translocating from cytoplasm to mitochondria decreased (Fig. 5B, C). Tests of cell proliferation ability using the CCK-8 assay, the result further showed that CsA $(5 \mu \mathrm{M})$ reduced the inhibitory effect of sesamin on A549 cell proliferation (Fig. 5A).

\section{DISCUSSION}

Sesamin, a type of lipid-soluble lignin originally isolated from sesame seeds, has since been found in more than 30 different plants, including willow, pepper, and magnolia [18]. A number of studies have shown that sesamin possesses bioactive properties, including anti-inflammatory and anticancer activity [3]. In 2010, Wang and colleagues reported that obtusilactone A and (-)-sesamin induce apoptosis in human lung cancer cells by inhibiting the mitochondrial protease Lon and activating DNA damage checkpoints [7]. A more recent study showed that sesamin blocks the PI3K/pAKT signaling pathway in lung cancer by downregulating the expression of COX2, leading to cell cycle arrest and induction of apoptosis [5]. In the current study, we found that sesamin inhibited the proliferation and migration of human lung cancer cell and induced oxidative stress in lung cancer cells, leading to a decline in mitochondrial membrane potential and subsequent induction of mitophagy and apoptosis.

ROS and antioxidants are key signaling molecules that participate in oxidative stress responses. Under specific pathological conditions, accumulation of excessive ROS can disrupt cellular homeostasis, leading to oxidative stress and mitochondrial dysfunction [19]. Mitochondria are the main sites of ROS production as well as targets of ROS. Mitochondria in cancer cells are both 
A
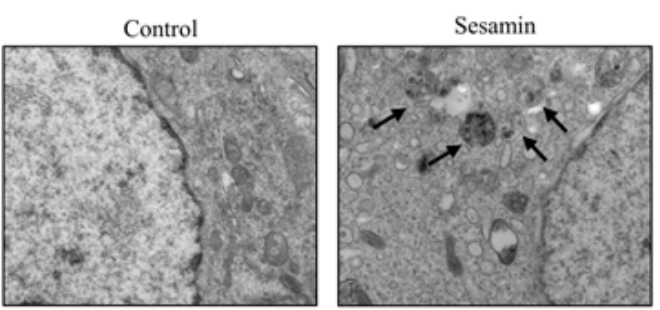

B

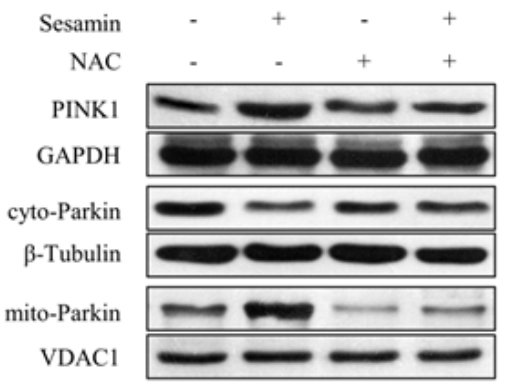

C

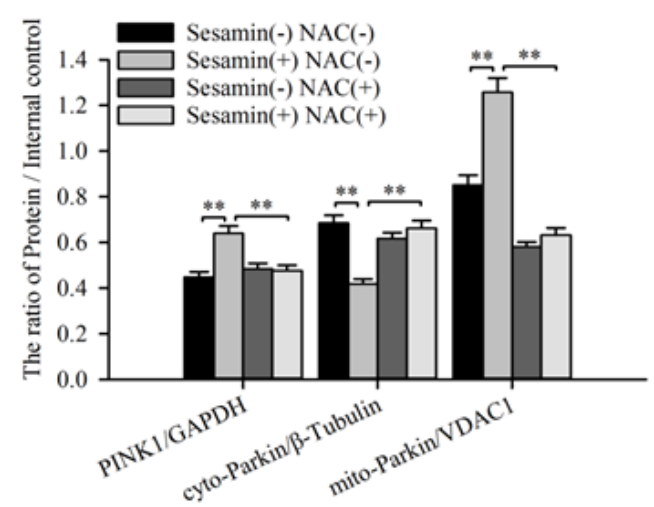

Fig. 4. Sesamin triggers mitophagy through a reactive oxygen species (ROS)-mediated PINK1/Parkin pathway in A549 cells. (A) A549 cells were treated with $40 \mu \mathrm{M}$ sesamin for $48 \mathrm{~h}$ and then observed by TEM (5,000x; arrows indicate autophagosomes). (B) Expression of PINK1 in whole cell and Parkin in cytoplasm or mitochondria of A549 cells treated with $40 \mu \mathrm{M}$ sesamin and/or $5 \mathrm{mM}$ NAC for $48 \mathrm{~h}$ was assessed by Western blotting with anti-PINK1, anti-Parkin, anti-VDAC1 (mitochondria marker), and anti- $\beta$-Tubulin (cytoplasm marker) antibodies. (C) Quantification of PINK1 and Parkin. Data are means \pm standard deviation $\left(n=3 ;{ }^{* *} \mathrm{p}<0.01\right)$. NAC, N-acetyl-L-cysteine.

structurally and functionally distinct from those in normal cells, and actively participate in metabolic recombination, characterized by ROS overproduction, to promote cancer development [20]. Therefore, reducing intracellular ROS levels and weakening the survival advantage that they confer may represent an effective strategy for inhibiting tumor development. In addition, excessive levels of ROS cause serious damage to cellular components, including DNA, proteins, and mitochondrial lipid membranes [21]. Data presented here indicate that sesamin induces alterations in the antioxidant system that lead to elevated ROS levels in A549 cells.

Mitochondria are the main source of ROS, which in turn af-
A

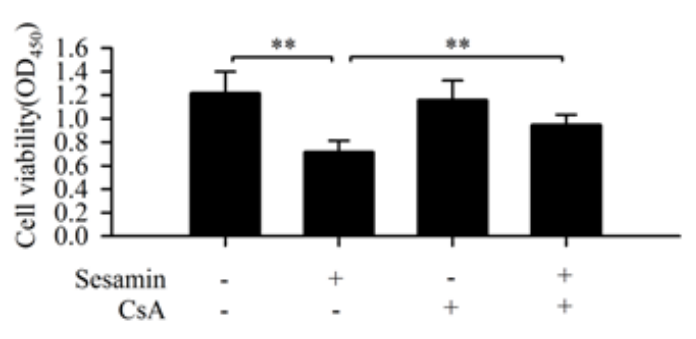

B

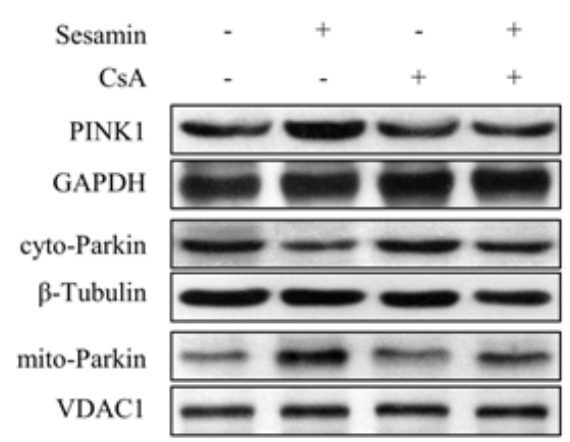

C

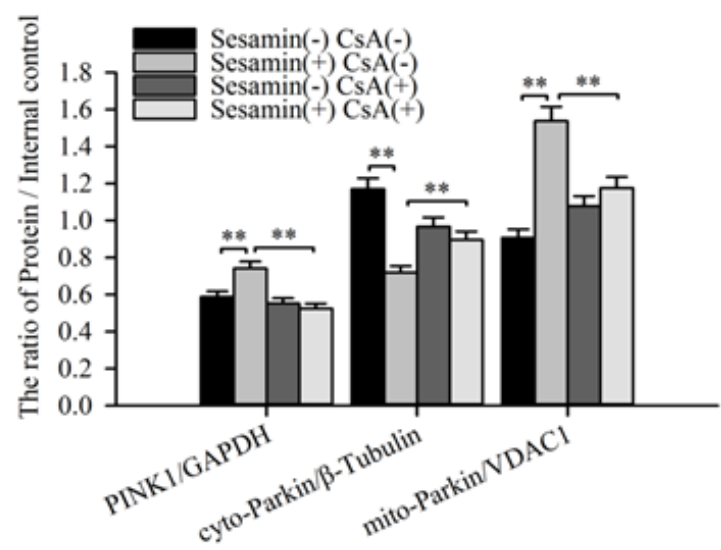

Fig. 5. Inhibition of mitophagy attenuates the inhibitory effect of sesamin on A549 cell viability. (A) A549 cells were treated with $40 \mu \mathrm{M}$ sesamin and/or $5 \mu \mathrm{M}$ cyclosporine A (CsA) for $48 \mathrm{~h}$, and cell viability was measured by CCK-8 assay. Data are means \pm standard deviation (SD) $\left(\mathrm{n}=3 ;{ }^{* *} \mathrm{p}<0.01\right)$. (B) A549 cells were treated with $40 \mu \mathrm{M}$ sesamin and/ or $5 \mu \mathrm{M}$ CsA for $48 \mathrm{~h}$, and then expression of PINK1 in whole cell and Parkin in cytoplasm or mitochondria was assessed by Western blotting with anti-PINK1, anti-Parkin, anti-VDAC1 (mitochondria marker), and anti- $\beta$-Tubulin (cytoplasm marker) antibodies. (C) Quantification of PINK1 and Parkin. Data are means \pm SD $\left(n=3 ;{ }^{* *} p<0.01\right)$.

fect mitochondria by causing a breakdown in the mitochondrial membrane potential $(\Delta \psi \mathrm{m})$. A decrease in $\Delta \psi \mathrm{m}$ is considered the earliest event in the apoptosis cascade. Following substantial changes in $\Delta \psi \mathrm{m}$, members of the caspase protease family are activated, initiating an apoptotic cascade reaction and irreversible entry into the apoptotic pathway [22]. Measurement of $\Delta \psi \mathrm{m}$ in A549 cells using the JC-1 assay kit showed that sesamin disrupts $\Delta \psi \mathrm{m}$, an effect that was attenuated by the antioxidant, NAC. These data indicate that sesamin triggers oxidative stress in A549 
cells, resulting in a decrease in $\Delta \psi \mathrm{m}$. In addition, measurements of apoptosis using flow cytometry and TUNEL assays showed that sesamin increased A549 cell apoptosis via a ROS-dependent mechanism.

Caspase- 3 is present in cells as an inactive precursor under normal circumstances, but is cleaved under stress conditions to generate an active fragment that mediates apoptosis [23]. Cellular apoptosis can be divided into two main pathways: endogenous and exogenous. Exogenous apoptosis is mediated by cell death receptor-cleaved caspase- 8 , whereas endogenous apoptosis is mediated by cleaved caspase-9 [24]. Excessive accumulation of ROS promotes mitochondrial membrane permeability and collapse of the mitochondrial membrane potential. Cytochrome c, an important electron transport component of the respiratory chain, is subsequently released [25], and in turn activates caspase-9, which activates the caspase cascade, and ultimately caspase- 3 , to induce apoptosis [26]. Experiments from the present study showed that sesamin treatment promoted upregulation of cytochrome $c$ and activation of caspase-3 and caspase-9. Notably, the increase in these proteins was blocked by pretreatment with NAC. Collectively, these findings indicate that sesamin induces apoptosis via the ROS-dependent intrinsic mitochondrial pathway.

$\mathrm{Bcl}-2$ is a crucial cancer-related protein in apoptosis research. Bcl-2 inhibits cell death induced by a variety of cytotoxic factors. The earlier discovery that overexpression of Bcl-2 enhances the resistance of cells to most cytotoxins led to the hypothesis that various apoptotic signaling mechanisms have a common pathway or intersection point that is regulated by Bcl-2 [27]. Excessive Bcl2 expression is associated with reduced generation of ROS. Previous studies support an indirect antioxidant effect of Bcl-2, such that $\mathrm{Bcl}-2$ inhibits the production of superoxide anions rather than directly scavenging ROS. In addition, decreasing Bcl-2 levels alters the mitochondrial permeability transition pore structure and increases mitochondrial membrane permeability, causing breakdown of the mitochondrial membrane potential [28]. Bax, which also belongs to the Bcl-2 family, is the most important proapoptotic protein in the human body and exerts an inhibitory effect on Bcl-2. The Bax/Bcl-2 ratio is regarded as a key factor in determining whether cells enter the apoptotic pathway [29]. Our Western blot data confirmed that sesamin increases the Bax/Bcl2 ratio. Taken together with previous experimental findings, our results suggest that sesamin induces A549 cell apoptosis via a ROS-dependent mitochondrial pathway.

Mitophagy is a type of autophagy process in which redundant and damaged mitochondria are selectively degraded, a concept first formally proposed by Lemasters [12] in 2005. During mitophagy, damaged mitochondria become isolated. Mitochondrial autophagocyte-related proteins are subsequently activated and accumulate on the surfaces of damaged mitochondria-specifically identifiable by the autophagosome membrane-and become encapsulated to form the mitochondrial autophagosome, which then fuses with the lysosome to form a mitochondrial autophago- some that mediates the degradation of damaged mitochondria [30]. Morphological changes in mitochondrial ultrastructure can be observed by TEM - the gold standard for the detection of mitochondrial autophagy. Using TEM to detect changes in mitochondrial ultrastructure in sesamin-treated A549 cells treatment, we found that a vacuolar double-layer membrane structure appeared around mitochondria, encapsulating them to form mitochondrial autophagosomes. These results indicate that sesamin induces mitophagy in A549 cells.

The PINK1/Parkin pathway has been identified as a paradigmatic mechanism for mammalian mitophagy. Under physiological conditions, dimeric PINK1 rapidly dissociates and is degraded in the intima after entering mitochondria, where it is maintained at a stable low level [31]. Studies have shown that, in the context of oxidative stress, PINK1 rapidly senses decreases in mitochondrial membrane potential characteristic of damaged mitochondria, and accumulates on the surface of the outer membrane of these mitochondria, where it induces accumulation of Parkin [32]. In the present study, TEM revealed the presence of many autophagosomes containing mitochondria in sesamin-treated A549 cells, suggesting that sesamin induces A549 cell mitophagy. We also found that sesamin increased the expression of PINK1 and induced the translocation of Parkin from cytoplasm to mitochondria. These effects were significantly attenuated by NAC, indicating that sesamin triggers mitophagy through a ROS-mediated PINK1/Parkin pathway in A549 cells.

Autophagic lysosomes selectively remove damaged, folded and redundant mitochondria, so as to maintain a balance between mitochondrial quality and quantity, and thus ensuring cell survival [33]. Thus, mitochondrial autophagy is generally thought to be a cell-protective mechanism. However, extensive autophagy also causes cytotoxic effects, leading to cell death [34]. Cyclosporin A is a specific inhibitor of mitochondrial permeability transport pathway, which plays a role by inhibiting the opening of mitochondrial permeability transport channels, and ultimately inhibits the cell apoptosis and necrosis dependent on mitochondrial permeability transport channels $[35,36]$. To explore the role of mitophagy in the effect of sesamin on A549 cells, we used CsA to block mitochondrial autophagy. We found that CsA diminished the cytotoxicity of sesamin toward A549 cells, indicating that sesamin induces A549 cell autophagic death.

\section{ACKNOWLEDGEMENTS}

This research was supported by grants from the National Natural Science Foundation of China (No. 31760330; No. 81860461); Natural Science Research Foundation of Jilin Province for Sciences and Technology (20190201240JC). 


\section{CONFLICTS OF INTEREST}

The authors declare no conflicts of interest.

\section{REFERENCES}

1. Ferlay J, Shin HR, Bray F, Forman D, Mathers C, Parkin DM. Estimates of worldwide burden of cancer in 2008: GLOBOCAN 2008. Int J Cancer. 2010;127:2893-2917.

2. Wakelee H, Kelly K, Edelman MJ. 50 Years of progress in the systemic therapy of non-small cell lung cancer. Am Soc Clin Oncol Educ Book. 2014:177-189.

3. Majdalawieh AF, Massri M, Nasrallah GK. A comprehensive review on the anti-cancer properties and mechanisms of action of sesamin, a lignan in sesame seeds (Sesamum indicum). Eur J Pharmacol. 2017;815:512-521.

4. Xu P, Cai F, Liu X, Guo L. Sesamin inhibits lipopolysaccharideinduced proliferation and invasion through the p38-MAPK and NF- $\kappa \mathrm{B}$ signaling pathways in prostate cancer cells. Oncol Rep. 2015;33:3117-3123.

5. Siao AC, Hou CW, Kao YH, Jeng KC. Effect of sesamin on apoptosis and cell cycle arrest in human breast cancer mcf-7 cells. Asian Pac J Cancer Prev. 2015;16:3779-3783.

6. Dou H, Yang S, Hu Y, Xu D, Liu L, Li X. Sesamin induces ER stressmediated apoptosis and activates autophagy in cervical cancer cells. Life Sci. 2018;200:87-93.

7. Wang HM, Cheng KC, Lin CJ, Hsu SW, Fang WC, Hsu TF, Chiu CC, Chang HW, Hsu CH, Lee AY. Obtusilactone A and (-)-sesamin induce apoptosis in human lung cancer cells by inhibiting mitochondrial Lon protease and activating DNA damage checkpoints. Cancer Sci. 2010;101:2612-2620.

8. Fang Q, Zhu Y, Wang Q, Song M, Gao G, Zhou Z. Suppression of cyclooxygenase 2 increases chemosensitivity to sesamin through the Akt-PI3K signaling pathway in lung cancer cells. Int J Mol Med. 2019;43:507-516.

9. Chen Y, Li H, Zhang W, Qi W, Lu C, Huang H, Yang Z, Liu B, Zhang L. Sesamin suppresses NSCLC cell proliferation and induces apoptosis via Akt/p53 pathway. Toxicol Appl Pharmacol. 2020;387:114848.

10. Chance B, Sies H, Boveris A. Hydroperoxide metabolism in mammalian organs. Physiol Rev. 1979;59:527-605.

11. Dawson TL, Gores GJ, Nieminen AL, Herman B, Lemasters JJ. Mitochondria as a source of reactive oxygen species during reductive stress in rat hepatocytes. Am J Physiol. 1993;264(4 Pt 1):C961-C967.

12. Lemasters JJ. Selective mitochondrial autophagy, or mitophagy, as a targeted defense against oxidative stress, mitochondrial dysfunction, and aging. Rejuvenation Res. 2005;8:3-5.

13. Xiao B, Deng X, Lim GGY, Zhou W, Saw WT, Zhou ZD, Lim KL, Tan EK. p62-Mediated mitochondrial clustering attenuates apoptosis induced by mitochondrial depolarization. Biochim Biophys Acta Mol Cell Res. 2017;1864:1308-1317.

14. Matsuda N, Sato S, Shiba K, Okatsu K, Saisho K, Gautier CA, Sou YS, Saiki S, Kawajiri S, Sato F, Kimura M, Komatsu M, Hattori N, Tanaka K. PINK1 stabilized by mitochondrial depolarization recruits Parkin to damaged mitochondria and activates latent Parkin for mitophagy. J Cell Biol. 2010;189:211-221.

15. Narendra D, Tanaka A, Suen DF, Youle RJ. Parkin is recruited selectively to impaired mitochondria and promotes their autophagy. $J$ Cell Biol. 2008;183:795-803.

16. Narendra DP, Jin SM, Tanaka A, Suen DF, Gautier CA, Shen J, Cookson MR, Youle RJ. PINK1 is selectively stabilized on impaired mitochondria to activate Parkin. PLoS Biol. 2010;8:e1000298.

17. Halestrap AP, Connern CP, Griffiths EJ, Kerr PM. Cyclosporin A binding to mitochondrial cyclophilin inhibits the permeability transition pore and protects hearts from ischaemia/reperfusion injury. Mol Cell Biochem. 1997;174:167-172.

18. Akl MR, Ayoub NM, Abuasal BS, Kaddoumi A, Sylvester PW. Sesamin synergistically potentiates the anticancer effects of $\gamma$-tocotrienol in mammary cancer cell lines. Fitoterapia. 2013;84:347-359.

19. Li L, Tan J, Miao Y, Lei P, Zhang Q. ROS and autophagy: interactions and molecular regulatory mechanisms. Cell Mol Neurobiol. 2015;35:615-621.

20. Yang Y, Karakhanova S, Hartwig W, D'Haese JG, Philippov PP, Werner J, Bazhin AV. Mitochondria and mitochondrial ROS in cancer: novel targets for anticancer therapy. J Cell Physiol. 2016;231:2570-2581.

21. Kang D, Hamasaki N. Mitochondrial oxidative stress and mitochondrial DNA. Clin Chem Lab Med. 2003;41:1281-1288.

22. Zhang B, Bian W, Pal A, He Y. Macrophage apoptosis induced by aqueous C60 aggregates changing the mitochondrial membrane potential. Environ Toxicol Pharmacol. 2015;39:237-246.

23. Vagner T, Mouravlev A, Young D. A novel bicistronic sensor vector for detecting caspase-3 activation. J Pharmacol Toxicol Methods. 2015;72:11-18.

24. Farias-de-Oliveira DA, Villa-Verde DM, Nunes Panzenhagen PH, Silva dos Santos D, Berbert LR, Savino W, de Meis J. Caspase-8 and caspase- 9 mediate thymocyte apoptosis in Trypanosoma cruzi acutely infected mice. J Leukoc Biol. 2013;93:227-234.

25. Martínez-Fábregas J, Díaz-Moreno I, González-Arzola K, Janocha S, Navarro JA, Hervás M, Bernhardt R, Velázquez-Campoy A, DíazQuintana A, De la Rosa MA. Structural and functional analysis of novel human cytochrome $\mathrm{C}$ targets in apoptosis. Mol Cell Proteomics. 2014;13:1439-1456.

26. Li J, Wu DD, Zhang JX, Wang J, Ma JJ, Hu X, Dong WG. Mitochondrial pathway mediated by reactive oxygen species involvement in $\alpha$-hederin-induced apoptosis in hepatocellular carcinoma cells. World J Gastroenterol. 2018;24:1901-1910.

27. Laulier C, Lopez BS. The secret life of Bcl-2: apoptosis-independent inhibition of DNA repair by Bcl-2 family members. Mutat Res. 2012;751:247-257.

28. Chen Q, Lesnefsky EJ. Blockade of electron transport during ischemia preserves bcl-2 and inhibits opening of the mitochondrial permeability transition pore. FEBS Lett. 2011;585:921-926.

29. Pan LL, Wang AY, Huang YQ, Luo Y, Ling M. Mangiferin induces apoptosis by regulating Bcl-2 and Bax expression in the CNE2 nasopharyngeal carcinoma cell line. Asian Pac J Cancer Prev. 2014;15:7065-7068.

30. Youle RJ, Narendra DP. Mechanisms of mitophagy. Nat Rev Mol Cell Biol. 2011;12:9-14.

31. Bertolin G, Ferrando-Miguel R, Jacoupy M, Traver S, Grenier K, Greene AW, Dauphin A, Waharte F, Bayot A, Salamero J, Lombès A, Bulteau AL, Fon EA, Brice A, Corti O. The TOMM machinery is a 
molecular switch in PINK1 and PARK2/PARKIN-dependent mitochondrial clearance. Autophagy. 2013;9:1801-1817.

32. Jin SM, Youle RJ. PINK1- and Parkin-mediated mitophagy at a glance. J Cell Sci. 2012;125(Pt 4):795-799.

33. Altshuler-Keylin S, Shinoda K, Hasegawa Y, Ikeda K, Hong H, Kang Q, Yang Y, Perera RM, Debnath J, Kajimura S. Beige adipocyte maintenance is regulated by autophagy-induced mitochondrial clearance. Cell Metab. 2016;24:402-419.

34. Kimura T, Takabatake Y, Takahashi A, Isaka Y. Chloroquine in cancer therapy: a double-edged sword of autophagy. Cancer Res. 2013;73:3-7.

35. Kim JS, He L, Lemasters JJ. Mitochondrial permeability transition: a common pathway to necrosis and apoptosis. Biochem Biophys Res Commun. 2003;304:463-470.

36. Kim JS, Qian T, Lemasters JJ. Mitochondrial permeability transition in the switch from necrotic to apoptotic cell death in ischemic rat hepatocytes. Gastroenterology. 2003;124:494-503. 